\title{
Hypothalamic Hamartomas: Two Cases
}

\author{
Tushar Kalekar \\ Department of Radio Diagnosis, D.Y. Patil Medical College, Pune, India \\ Email: dr.tushar.kalekar@gmail.com
}

Received 20 February 2015; accepted 9 March 2015; published 11 March 2015

Copyright (C) 2015 by author and Scientific Research Publishing Inc.

This work is licensed under the Creative Commons Attribution International License (CC BY).

http://creativecommons.org/licenses/by/4.0/

(c) (i) Open Access

\begin{abstract}
This is a MRI study of two patients presented with history of gelastic seizures since many years ago. Plain and post intravenous gadolinium multiplanar MRI imaging of the brain is performed. It showed well-defined non-enhancing mass lesions in the region of hypothalamus and tuber cinereum. It showed signal intensity similar to the gray matter and imaging diagnosis of hypothalamic hamartoma is made.
\end{abstract}

\section{Keywords}

\section{Hamartoma, Hypothalamus, MR Imaging}

\section{Introduction}

Hypothalamic hamartomas, rare lesions of the floor of the third ventricle, are manifested with a spectrum of clinical symptoms that include precocious puberty, gelastic seizures, and behavioral disorders. Hypothalamic hamartomas represent neoplastic proliferation of well-organized brain tissue similar to heteropias [1]. Classical location and magnetic resonance imaging (MRI) features strongly favor the diagnosis.

As these lesions are usually small in size and isointense signal as compared to adjacent gray matter of hypothalamus, they can be easily missed on routine MRI imaging. High resolution MRI sequences with special attention in the region of hypothalamus should be performed whenever scanning patients with these types of seizures or associated precocious puberty. MRI is the most important modality for the diagnosis of this entity.

\section{Case Reports}

\subsection{Case 1}

A 19-year-old boy presented with episodes of epileptic laughter (gelastic seizures) 2 to 3 times a day since ten years old.

Clinical examination of the patient was normal. There was no focal neurological deficit. EEG study was 
within normal limits. Laboratory studies and endocrine studies were normal.

Multiplanar multiecho MRI study was performed on 1.5 T GE scanner with high resolution, thin coronal and sagittal images were performed in the region of thalamus and this was followed by post intravenous gadolinium study. MRI showed a well-circumscribed mass lesion in the region of hypothalamus (Figure 1 and Figure 2). Coronal and sagital scans demonstrated contiguity between the mass and the tuber cinereum. It appeared isointense to the cortex on T1W and T2W images with no post contrast enhancement (Figure 3). In view of the clinical findings and imaging findings on MR, a diagnosis of hypothalamic hamartoma was considered. Patient was started on medroxy progesterone acetate.

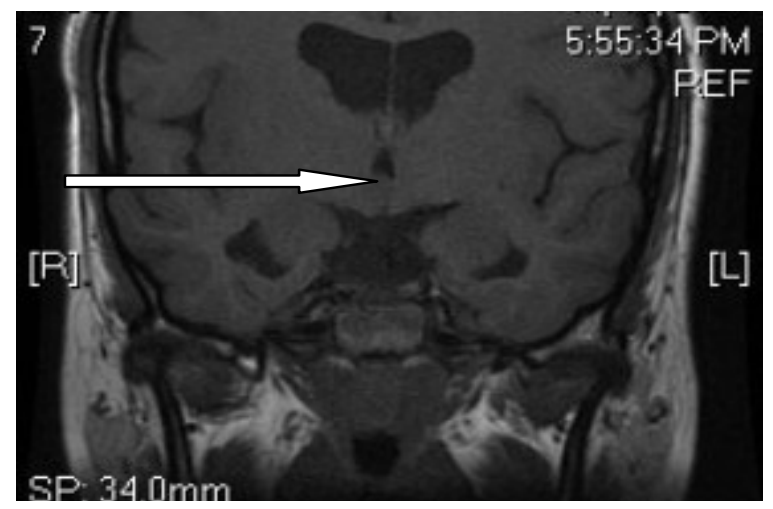

Figure 1. Plain T1W coronal image showing a small well-defined focal mass lesion in the region of hypothalamus on right side appearing isointense to cortex.

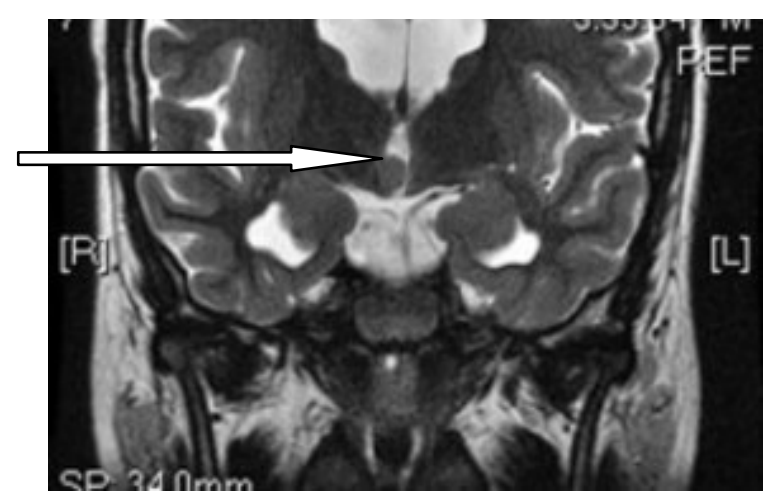

Figure 2. Plain T2 WI coronal image showing a small well-defined focal mass lesion in the region of hypothalamus on right side appearing isointense to cortex.

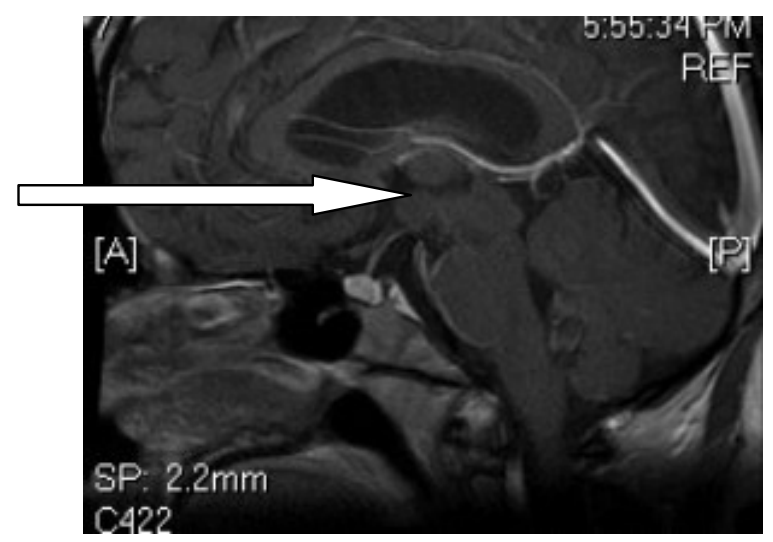

Figure 3. Post gadolinium T1W sagittal image showing a small well-defined focal mass lesion in the region of hypothalamus and tuber cinereum showing no enhancement. 


\subsection{Case 2}

A 17-year-old girl presented with headache and repeated episodes of gelastic seizures since four years ago. Clinical examination of the patient was normal. There was no focal neurological deficit. EEG study was within normal limits. Laboratory studies and endocrine studies were normal.

Multiplanar multiecho MRI study was performed on 1.5 T GE scanner with high resolution, thin coronal and sagittal images were performed in the region of thalamus and this was followed by post intravenous gadolinium study.

There was a well defined sessile focal lesion in the hypothalamic region (Figure 4) and in the floor of third ventricle on right side with involvement of the tuber cinereum. It appeared isointense to the cortex on T1W and T2W images with no post contrast enhancement (Figure 5). There was mild dilation of both the lateral ventricles. Imaging diagnosis of hypothalamic hamartoma was made and patient started on progesterone treatment.

\section{Discussion}

A hamartoma of the tuber cinereum or hypothalamus is a congenital malformation consisting of neuronal tissue in an ectopic location. Histologically, this lesion is often similar to the adjacent hypothalamus, although other neuronal tissues may be included. These lesions may be quite large at presentation and may be associated with other congenital or neuronal migration abnormalities. Most of these lesions occur in hypothalamus. Other locations may be the subcortical cerebral cortex and periventricular region.

Hypothalamic hamartomas are small pedunculated growths and contiguous with posterior hypothalamus between the tuber cinereum and mamillary bodies and they can extend between the optic chiasm and pons and usually do not distort the hypothalamus or other parts of the base of the brain unless they are very large.

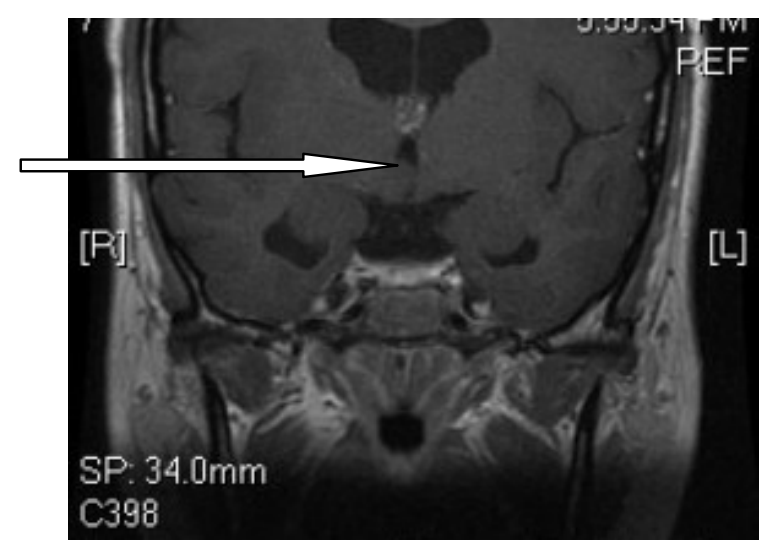

Figure 4. Post gadolinium T1W coronal image showing a small well-defined non enhancing isointense focal mass lesion in the region of hypothalamus.

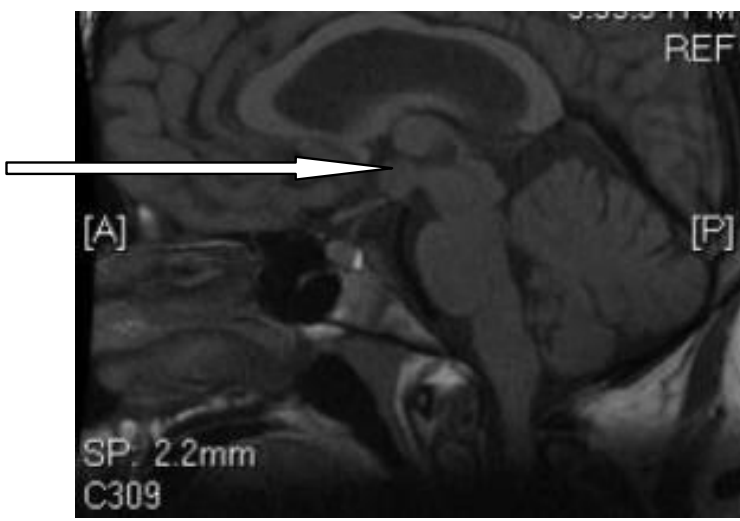

Figure 5. Plain T1 WI sagittal image showing a small well-defined focal mass lesion in the region of hypothalamus and tuber cinereum appearing isointense to cortex. 
They occur with equal frequency in males and females [2]. Most patients usually present in the first or second decade of life. Two common clinical presentations include 1) isosexual precocious puberty [3]; 2) less often, neurological signs including generalized or partial complex seizures accompanied by associated impaired intellect and/or behavioral disorders. Hypothalamic hamartoma is the commonest tumoral cause of precocious puberty [4]. Gelastic (laughing or grimacing) seizures are particularly characteristic of hypothalamic hamartoma. Isosexual precocious puberty may be from premature disappearance of normal inhibitory factors on hypothalamus, which results in luteinising hormone releasing factor (LHRH) being released prematurely thus activating the pituitary gonadal axis. Larger hamartomas are less likely to produce precocious puberty [2]. Occasionally, visual disturbances may be present because of involvement of optic pathways.

On imaging, hypothalamic hamartomas produce characteristic soft tissue masses isointense to grey matter. They are homogeneous and sharply marginated by the surrounding CSF, as they do not disrupt the blood brain barrier; there is no enhancement on post-contrast images. Calcification is rare and hemorrhage is not described in these lesions. The anatomic location of these hamartomas together with signal intensity similar to grey matter on T1 WI and a higher intensity than gay matter on PDWI and T2 WI strongly supports this diagnosis [5]. Our patient showed classical well-marginated soft tissue masses of grey matter intensity in region of hypothalamus. The diagnosis was made on the basis of the gelastic seizures, characteristic location and isointensity to normal brain.

Few congenital malformations are described in association with hypothalamic hamartoma, such as duplication of the pituitary gland and infundibular stalk, persistence of craniopharyngeal canal which a small bony defect in the skull base which connects sella turcica to the nasopharynx and basal cephaloceles [4].

Differential diagnosis of hypothalamic hamartoma may include craniopharyngioma, optic gliomas, hypothalamic gliomas and gangliogliomas. Calcification is common in craniopharyngiomas and optic gliomas. Anatomically, the hypothalamus forms the floor of the third ventricle and is bounded anteriorly by the optic chiasm and posteriorly by the mammillary bodies. The most common masses arising in this region are hypothalamic or optic pathway gliomas which expand and infiltrate the hypothalamus and adjacent structures. In hypothalamic hamartoma, however, the chiasm is intact, and the mass extends exophytically from the hypothalamus with little distortion of the normal tissues.

They are benign lesions and typically to do not grow, however, symptomatology can be progressive. Children with central precocious puberty can be treated medically.

Intractable epilepsy can respond to surgery (resection or disconnection, depending on the morphology of the tumour) and this can be performed via an open craniotomy or more recently via an endoscope. Gamma knife radio surgery has also been used.

Medical treatment with medroxy progesterone acetate is the treatment of choice in most patients [6]. Followup MRI is recommended every 6 to 12 months for demonstrating lack of growth.

As in both of these cases, MRI findings were classical and no other differential diagnosis was made. Patient was started on medical treatment with medroxy progesterone acetate. There was symptomatic relief and showed reduced frequency and duration of seizure attacks. We recommended six-month MRI follow-up for assessment of interval change in size of the hamartoma.

In conclusion, hypothalamic hamartomas are rare congenital malformations. Classical clinical presentation of gelastic seizures with MR imaging features of isointense non-enhancing mass of the grey matter intensity in the region of the hypothalamus is highly suggestive of the diagnosis.

\section{References}

[1] Le Marquand, H.S. and Russell, D.S. (1934) A Case of Pubertas Praecox in a Boy Associated with a Tumor in the Floor of the Third Ventricle. Berk Hosp Rep., 3, 31-61.

[2] Hubbard, A.M. and Egelhoff, J.C. (1989) MRI Imaging of Large Hypothalamic Hamartomas of Two Infants. American Journal of Neuroradiology, 10, 1277-1279.

[3] Reith, K.G., Comite, F., Dwyer, A.I., Nelson, M.J., Pescovitz, O., Shawker, T.H., et al. (1987) CT of Cerebral Abnormalities in Precocious Puberty. American Journal of Neuroradiology, 8, 283-290.

[4] Kizikilic, O., Yalcin, O., Yeldirim, T., Sener, L., Parmaksiz, G. and Erdogan, B. (2005) Hypothalamic Hamartoma Associated with Craniopharyngeal Canal. American Journal of Neuroradiology, 26, 65-67

[5] Boyko, O.B., Curnes, J.T., Oakes, W. and Burger, P.C. (1991) Hamartomas of the Tuber Cinereum: CT, MR, and Pathologic Findings. American Journal of Neuroradiology, 12, 309-314.

[6] Hochma, H.I., Judge, D.M. and Reichlin, S. (1980) Precocious Puberty and Hypothalamic Hamartoma. Pediatrics, 67, 236-244. 